\title{
Identificação Inversa Recursiva Multivariável Baseada em Modelo Nebuloso de Realização Mínima no Espaço de Estados com Observador de Kalman
}

\author{
Adriano Mendes Magalhães* \\ Ginalber Luiz de Oliveira Serra** \\ ${ }^{*}$ Universidade Federal do Maranhão, Av. do Português, Vila Bacanga, \\ s.n., São Luis, MA, Brasil (e-mail: \\ adriano.mendes.magalhaes@gmail.com) \\ ** Instituto Federal de Educação, Ciência e Tecnologia do Maranhão, \\ Av. Getúlio Vargas, Monte Castelo, s.n., São Luis, MA, Brasil \\ (e-mail: ginalber@ifma.edu.br)
}

\begin{abstract}
In this paper, a methodology for inverse fuzzy modeling of multivariable nonlinear dynamic systems, by direct approach, with state space minimal realization, from experimental data, is proposed. The adopted methodology consists of a formulation for online parametric estimation of the inverse fuzzy model, based on the fuzzy and recursive version of the Observer/Kalman Identification algorithm, using the approach of the Gustafson-Kessel fuzzy clustering algorithm. The analysis of the experimental results related to the recursive identification of Helicopter 2DoF illustrate the efficiency and applicability of the adopted methodology.

Resumo: Neste artigo, uma metodologia para modelagem nebulosa inversa de sistemas não lineares multivariáveis, pela abordagem direta, de realização mínima no espaço de estados, a partir de dados experimentais é proposta. A metodologia adotada consiste numa formulação para estimação paramétrica online do modelo nebuloso inverso, baseada na versão nebulosa e recursiva do algoritmo OKID (Observer/Kalman Identification), usando-se a abordagem do algoritmo de agrupamento nebuloso Gustafson-Kessel. A análise dos resultados experimentais relacionados à identificação recursiva de um Helicópitero 2DoF ilustram a eficiência e aplicabilidade da metodologia adotada.
\end{abstract}

Keywords: System inverse identification; multivariable dynamic systems; Kalman observer; minimal realization; fuzzy inference system.

Palavras-chaves: Identificação inversa de sistemas; sistemas dinâmicos multivariáveis; observador de Kalman; realização mínima; sistema de inferência nebuloso.

\section{INTRODUÇÃO}

Para identificação inversa de sistemas dinâmicos, destacamse três abordagens principais: abordagem direta (contexto de estudo neste artigo), abordagem indireta e aprendizagem especializada (Sousa and Kaymak, 2002; Jung and Enqvist, 2013). Na abordagem direta, o modelo inverso é obtido em uma só etapa, através dos dados de saída e entrada do sistema dinâmico a ser identificado. Alinhado a isso, a combinação da teoria de modelagem inversa com técnicas de inteligência computacional, especificamente sistemas nebulosos, tem apresentado resultados promissores (Lee and Liu, 2014; Lai et al., 2017; Rahbari et al., 2018). Em Boukezzoula et al. (2003), uma metodologia de inversão de modelo nebuloso Takagi-Sugeno é proposta como estratégia de controle baseado em modelo interno de sistemas não lineares. Em Sun et al. (2009), um algoritmo que reúne as técnicas de SVM (do inglês Support Vector Machine) com mínimos quadrados e agrupamento nebuloso c-means é proposto para a identificação de sistemas dinâmicos baseada em modelos não lineares inversos. Além disso, para a identificação de sistemas não lineares MIMO (do inglês Multiple Input and Multiple Output) baseada em modelos nebulosos inversos, a teoria do espaço de estados se torna particularmente atrativa (Nelles, 2001; Aguirre, 2015). A propriedade de compensação paralela distribuída, inerente à teoria de sistemas nebulosos, permite que a proposição consequente seja representada por modelos lineares e invariantes com o tempo, realizando o mapeamento dinâmico inverso associado, respectivamente, a pontos de operação do sistema dinâmico a ser identificado, de modo que os modelos no espaço de estados sejam estabelecidos. Assim, neste artigo, é proposta uma metodologia para modelagem inversa de sistemas não lineares multivariáveis a partir de dados experimentais, pela abordagem direta, baseada em modelo nebuloso de realização mínima com estrutura Takagi-Sugeno, no espaço de estados.

A originalidade da metodologia proposta consiste na formulação para estimação paramétrica do modelo nebuloso inverso, a qual é baseada na versão nebulosa e recursiva 
do algoritmo OKID (Observer/Kalman Identification) em função do algoritmo de agrupamento nebuloso GustafsonKessel (Babuška, 1998), visando-se os seguintes aspectos de interesse:

- Janelamento de dados para implementação iterativa do algoritmo de agrupamento nebuloso GustafssonKessel;

- Estimação paramétrica no espaço de estados, baseada na versão nebulosa e recursiva do algoritmo OKID;

- Estimação de estados nebulosos no contexto do mapeamento inversos de sistemas dinâmicos não lineares.

\section{METODOLOGIA}

A metodologia adotada consiste na formulação matemática para identificação nebulosa inversa multivariável, no contexto do espaço de estados, usando-se a abordagem direta, a partir dos dados experimentais de entrada e saída.

Seja um sistema MIMO não linear de $r$ entradas $\mathbf{u}=$ $\left[u_{1}, \cdots, u_{r}\right]^{T} \in \mathbb{R}^{r \times 1}$ e $m$ saídas $\mathbf{y}=\left[y_{1}, \cdots, y_{m}\right]^{T} \in$ $\mathbb{R}^{m \times 1}$. Define-se a amostra do conjunto de dados o vetor $\mathbf{z}_{y u} \in \mathbb{R}^{(m+r) \times 1}$ tal que $\mathbf{z}_{y u} \triangleq\left[\mathbf{y}^{T}, \mathbf{u}^{T}\right]^{T} \in \mathbb{R}^{(m+r) \times 1}$. A priori, seja $\mathbf{u}=\mathbf{u}(k), \mathbf{y}=\mathbf{y}(k)$ e $\mathbf{z}_{y u}=\mathbf{z}_{y u}(k)$. Define-se a janela de dados deslizante sobre o tempo, como sendo a matriz $\mathbf{Z}_{y u}(k) \in \mathbb{R}^{(m+r) \times l}$, referente ao mapeamento da saída y para entrada $\mathbf{u}$, tal que:

$$
\mathbf{Z}_{y u}(k)=\left[\mathbf{z}_{y u}(k-l+1) \cdots \mathbf{z}_{y u}(k)\right]
$$

$\forall k \in \mathbb{Z}_{+}^{*} k \geq l$, onde $l$ é o número de amostras de um conjunto de dados iniciais. Assim se $k=l$, então tem-se $\mathbf{Z}_{y u}(l)$.

Define-se a base de regras, referente à classe de modelo nebuloso inverso adotada, dada por:

$$
\begin{gathered}
R^{i}: \operatorname{SE} \tilde{\mathbf{y}}(k) \text { é } \mathscr{Y} y u(k) \mathrm{E} \tilde{\mathbf{u}}(k) \text { é } \mathscr{U}_{y u}^{i}(k) \\
\text { ENTÃO }\left\{\begin{array}{r}
\hat{\mathbf{u}}^{i}(k)=\widehat{\boldsymbol{\Gamma}}^{i}(k) \hat{\mathbf{x}}_{y u}^{i}(k)+\widehat{\boldsymbol{\Delta}}^{i}(k) \mathbf{y}(k) \\
\hat{\mathbf{x}}_{y u}^{i}(k+1)=\widehat{\mathbf{G}}^{i}(k) \hat{\mathbf{x}}_{y u}^{i}(k)+\widehat{\mathbf{H}}^{i}(k) \mathbf{y}(k) \\
-\widehat{\mathbf{O}}^{i}(k)\left[\mathbf{u}(k)-\hat{\mathbf{u}}^{i}(k)\right]
\end{array}\right.
\end{gathered}
$$

onde $i \in\{1, \ldots, c\} \subset \mathbb{Z}_{+}^{*}$ é o indice da regra, do agrupamento ou do ponto de operação; $c \in \mathbb{Z}_{+}^{*}, c>1$ é o número de regras da base de regras. Os vetores $\hat{\mathbf{x}}_{y u}^{i}(k+1) \in \mathbb{R}^{n \times 1}$ e $\hat{\mathbf{u}}^{i}(k) \in \mathbb{R}^{r \times 1}$ definem respectivamente a equação de estados estimados e a equação de saídas estimadas do submodelo local (correspondente às entradas do sistema dinâmico real), cujos parâmetros $\widehat{\mathbf{G}}^{i} \in \mathbb{R}^{n \times n}, \widehat{\mathbf{H}}^{i} \in \mathbb{R}^{n \times m}$, $\widehat{\mathbf{O}}^{i} \in \mathbb{R}^{n \times r}, \widehat{\boldsymbol{\Gamma}}^{i} \in \mathbb{R}^{r \times n}$ e $\widehat{\boldsymbol{\Delta}}^{i} \in \mathbb{R}^{r \times m}$ são a matriz dinâmica ou matriz de estados, a matriz de entradas, a matriz do observador de estados, a matriz de saidas e matriz de transição direta, respectivamente.

Na proposição antecedente da base de regras, $\widetilde{\mathbf{u}}(k)$ e $\widetilde{\mathbf{y}}(k)$ são variáveis linguísticas das entradas e saídas, respectivamente, e $\mathscr{Y}_{y u}^{i}(k)$ e $\mathscr{U}_{y u}^{i}(k)$ são seus respectivos valores ou termos linguísticos, os quais são obtidos para cada ponto de operação ou regra $i$, e matematicamente são definidos por conjuntos nebulosos, cujos graus de pertinência são tais que $\mu_{\mathscr{Y} \mathscr{Y}^{i}(k)}(k) \triangleq \mu_{\mathscr{Y}}^{i u}(k)(\mathbf{y}(k)): \mathbb{R}^{m \times 1} \mapsto(0,1] \subset \mathbb{R}$ e $\mu_{\mathscr{U}_{y u}^{i}(k)}(k) \triangleq \mu_{\mathscr{U}_{y u}^{i}(k)}(\mathbf{u}(k)): \mathbb{R}^{r \times 1} \mapsto(0,1] \subset \mathbb{R}$. O grau de ativação normalizado da i-ésima regra $\gamma_{y u}^{i}(k) \in(0,1] \subset$ $\mathbb{R}$, é dado por:

$$
\gamma_{y u}^{i}(k) \triangleq \frac{\beta_{y u}^{i}(k)}{\sum_{i=1}^{c} \beta_{y u}^{i}(k)}
$$

onde $\beta_{y u}^{i}(k) \in(0,1] \subset \mathbb{R}$ é o grau de ativação da $i$ ésima regra na $k$-ésima amostra de dados. Na proposição consequente da base de regras, em cada ponto de operação $i$, um submodelo local inverso, no contexto do espaço de estados, com o ganho do observador de Kalman é estimado.

Como mecanismo ou máquina de inferência, inerente ao modelo nebuloso Takagi-Sugeno, definem-se o estado estimado nebuloso e a entrada estimada nebulosa do sistema como sendo os vetores $\widetilde{\hat{\mathbf{x}}}_{y u}=\tilde{\hat{\mathbf{x}}}_{y u}(k) \in \mathbb{R}^{n \times 1} \mathrm{e}$ $\tilde{\hat{\mathbf{u}}}=\tilde{\hat{\mathbf{u}}}(k) \in \mathbb{R}^{r \times 1}$, tais que

$$
\begin{array}{r}
\tilde{\hat{\mathbf{u}}}=\tilde{\hat{\mathbf{u}}}(k) \triangleq \sum_{i=1}^{c} \gamma_{y u}^{i}(k) \hat{\mathbf{u}}^{i}(k) \\
\tilde{\hat{\mathbf{x}}}_{y u}=\tilde{\hat{\mathbf{x}}}_{y u}(k) \triangleq \sum_{i=1}^{c} \gamma_{y u}^{i}(k) \hat{\mathbf{x}}_{y u}^{i}(k)
\end{array}
$$

\subsection{Estimação Paramétrica do Antecedente}

Na proposição antecedente, o grau de ativação normalizado $\gamma_{y u}^{i}(k)$ da $i$-ésima regra é o parâmetro a ser estimado para cada amostra de dados experimentais. Para isso, pode-se, por exemplo, executar o algoritmo de agrupamento de Gustafson-Kessel (GK) (Babuška et al., 2002) sobre uma janela (batelada) fixa de dados $\mathbf{Z}_{y u}(l)$, e obter o $\gamma_{y u}^{i}(k) \forall k \in[1, l]$, por aplicar a metodologia de projeção e extensão cilíndrica diretamente sobre os conjuntos nebulosos dos agrupamentos resultantes, conforme é proposto em (Magalhães and Serra, 2018). Porém nesse artigo, propõese obter o $\gamma_{y u}^{i}(k)$ diretamente da matriz correspondente à partição nebulosa dos agrupamentos, a partir da execução do algoritmo GK sobre a janela temporal de dados $\mathbf{Z}_{y u}(k)$ de (1), para todo $k \geq l$.

O algoritmo de agrupamento GK é definido de acordo com um procedimento iterativo de otimização da seguinte função custo (Babuška et al., 2002):

$$
J\left(\mathbf{Z} ; \mathfrak{R}, \mathfrak{C},\left\{\mathfrak{\Re}^{i}\right\}\right)=\sum_{i=1}^{c} \sum_{\xi=1}^{k}\left(\mu_{i \xi}\right)^{\varrho} \mathcal{D}_{i \xi \mathfrak{\Re}^{i}}^{2}
$$

onde $\mathbf{Z} \in \mathbb{R}^{\varsigma \times k}$ é a base de dados, dos quais serão agrupados; $\{c, \varsigma, k, \varrho\} \in \mathbb{Z}_{+}^{*}$ são respectivamente o número de agrupamentos, o número de observações (linhas), o número de amostras (colunas) da base de dados e o 
expoente de ponderação que determina a nebulosidade dos agrupamentos resultantes, respectivamente. A matriz $\mathfrak{R} \in \mathbb{R}^{c \times k}$ corresponde à partição nebulosa com os graus de pertinência das amostras de dados em relação aos núcleos ou centros dos agrupamentos $\mathfrak{c}_{i}^{(\ell)} \in \mathbb{R}^{\varsigma \times 1}$, dados por (Babuška, 1998):

$$
\mathbf{c}_{i}^{(\ell)}=\frac{\sum_{\xi=1}^{k}\left(\mu_{i \xi}^{(\ell-1)}\right)^{\varrho} \mathbf{z}(\xi)}{\sum_{\xi=1}^{k}\left(\mu_{i \xi}^{(\ell-1)}\right)^{\varrho}}
$$

onde $\ell$ é o índice do processo iterativo de otimização. O termo $\mathcal{D}_{i \xi \Re^{i}}^{2}$ é a distância quadrática de Mahalanobis entre a $\xi$-ésima amostra de $\mathbf{Z}$ e o $i$-ésimo centro de agrupamento $\mathfrak{c}_{i}$, de acordo com matriz de norma induzida $\mathfrak{\Re}^{i} \in \mathbb{R}^{\varsigma \times \varsigma}$, dada por (Babuška, 1998):

$$
\begin{aligned}
\mathfrak{\Re}^{i} & =\left[\nu_{i} \operatorname{det}\left(\mathbf{F}^{i}\right)\right]^{1 / \eta}\left(\mathbf{F}^{i}\right)^{-1} \\
\mathcal{D}_{i \xi \mathfrak{R}^{i}}^{2} & =\left(\mathbf{z}(\xi)-\mathfrak{c}_{i}^{(\ell)}\right)^{T} \mathfrak{\Re}^{i}\left(\mathbf{z}(\xi)-\mathfrak{c}_{i}^{(\ell)}\right)
\end{aligned}
$$

onde $\nu_{i} \in \mathbb{R}$ é o $i$-ésimo valor do volume (constante) do agrupamento e $\mathbf{z}(\xi) \in \mathbb{R}^{\varsigma \times 1}$ é o vetor da $\xi$-ésima amostra de dados de $\mathbf{Z}$. A matriz $\mathbf{F}^{i}$ corresponde à covariância nebulosa referente ao $i$-ésimo agrupamento, definida por (Babuška, 1998):

$$
\mathbf{F}^{i}=\frac{\sum_{\xi=1}^{k}\left(\mu_{i \xi}^{(\ell-1)}\right)^{\varrho}\left(\mathbf{z}(\xi)-\mathfrak{c}_{i}^{(\ell)}\right)\left(\mathbf{z}(\xi)-\mathfrak{c}_{i}^{(\ell)}\right)^{T}}{\sum_{\xi=1}^{k}\left(\mu_{i \xi}^{(\ell-1)}\right)^{\varrho}}
$$

a qual é simétrica, semipositiva e não singular (Aguirre, 2015).

A partir de (7) a (10), as $L$ iterações do processo de otimização são executadas até alcançar uma tolerância mínima $\vartheta \in \mathbb{R}_{+}^{*}$ do erro absoluto entre os elementos da matriz de partição nebulosa $\mathfrak{R}$, em um valor de iteração do ponto ótimo $\ell=\ell^{*}$. Essa matriz $\mathfrak{M}$ pode matematicamente ser definida como:

$$
\mathfrak{R}=\left[\mu_{i \xi}\right]_{c \times N} \triangleq\left[\begin{array}{ccccc}
\mu_{11} & \cdots & \mu_{1 \xi} & \cdots & \mu_{1 N} \\
\vdots & \ddots & \vdots & & \vdots \\
\mu_{i 1} & \cdots & \mu_{i \xi} & \cdots & \mu_{i N} \\
\vdots & & \vdots & \ddots & \vdots \\
\mu_{c 1} & \cdots & \mu_{c \xi} & \cdots & \mu_{c N}
\end{array}\right]
$$

com $\mu_{i \xi} \in(0,1] \subset \mathbb{R}$ sendo o grau de pertinência, o qual informa quanto cada amostra de dados $\xi$ pertence ao agrupamento $i$, tal que

$$
\mu_{i \xi}=\mu_{i \xi}^{\left(\ell^{*}\right)}=\frac{1}{\sum_{j=1}^{c}\left(\frac{\mathcal{D}_{i \xi \Re^{i}}^{2}}{\mathcal{D}_{j \xi \Re^{j}}^{2}}\right)^{1 /(\varrho-1)}}
$$

Por fim, fazendo-se $\mathbf{Z}=\mathbf{Z}_{y u}(k)$, tem-se $\varsigma=m+r$, e:

$$
\gamma_{y u}^{i}(k)=\mu_{i k}^{\left(\ell^{*}\right)}
$$

\subsection{Estimação Paramétrica da Proposição Consequente}

Na proposição consequente da base de regras, conforme em (2), as matrizes $\widehat{\mathbf{G}}^{i}(k), \widehat{\mathbf{H}}^{i}(k), \widehat{\mathbf{O}}^{i}(k), \widehat{\boldsymbol{\Gamma}}^{i}(k), \widehat{\boldsymbol{\Delta}}^{i}(k)$ são os parâmetros a serem estimados por meio de uma versão nebulosa e recursiva do algoritmo OKID.

\subsubsection{Estimação dos parâmetros de Markov na etapa re- cursiva}

Na etapa recursiva, o $i$-ésimo submodelo local inverso é desenvolvido no sentido dos mínimos quadrados ponderados recursivos com compensação paramétrica, dado por:

$$
\begin{aligned}
& \boldsymbol{\eta}^{i}(k-\tau)=\mathbf{u}(k-\tau)-\underline{\widehat{\mathbf{Y}}}_{y u}^{i}[k-(\tau+1)] \mathbf{v}_{y u}[k-(\tau+1)] \\
& \mathbf{\Xi}_{y u}^{i}[k-(\tau+1)]=\left\{\mathbf{v}_{y u}[k-(\tau+1)]\right\}^{T} \mathbf{P}_{y u}^{i}[k-(\tau+2)]
\end{aligned}
$$

$$
\begin{gathered}
\mathbf{K}_{y u}^{i}[k-(\tau+1)]= \\
\frac{\boldsymbol{\Xi}_{y u}^{i}[k-(\tau+1)]}{\left[\gamma_{y u}^{i}(k-\tau)\right]^{-1}+\boldsymbol{\Xi}_{y u}^{i}[k-(\tau+1)] \mathbf{v}_{y u}[k-(\tau+1)]}
\end{gathered}
$$

$$
\begin{gathered}
\mathbf{P}_{y u}^{i}[k-(\tau+1)]=\mathbf{P}_{y u}^{i}[k-(\tau+2)]\left\{\mathbf{I}_{\mathbf{P}_{y u}^{i}}-\right. \\
\left.\mathbf{v}_{y u}[k-(\tau+1)] \mathbf{K}_{y u}^{i}[k-(\tau+1)]\right\} \\
\underline{\widehat{\mathbf{Y}}}_{y u}^{i}(k)=\left[1-f_{T}\right] \underline{\widehat{\mathbf{Y}}}_{y u}^{i}(k-1)+f_{T}\left\{\underline{\widehat{\mathbf{Y}}}_{y u}^{i}[k-(\tau+1)]+\right. \\
\left.\boldsymbol{\eta}^{i}(k-\tau) \mathbf{K}_{y u}^{i}[k-(\tau+1)]\right\}
\end{gathered}
$$

onde $f_{T}=1 / T_{f} \in \mathbb{R}_{+}$. Os outros parâmetros de (14) a (18) são descritos como segue: $q \in \mathbb{Z}_{+}^{*}$ é o parâmetro de predição associado aos estados; $T_{f} \in \mathbb{Z}_{+}^{*}$ é a constante de suavização exponencial, $\tau \in \mathbb{Z}_{+}^{*}$ é a constante de atualização paramétrica descorrelacionada, definida como $\tau \triangleq n_{\min }, \boldsymbol{\eta}^{i}(k) \in \mathbb{R}^{r \times 1}$ é o vetor inovação, $\mathbf{K}_{y u}^{i}(k-$ $1) \in \mathbb{R}^{1 \times[m+q(m+r)]}$ é o vetor ganho de inovação; $\mathbf{P}_{y u}^{i}(k-$ 1) $\in \mathbb{R}^{[m+q(m+r)] \times[m+q(m+r)]}$ é a matriz de covariância de estimação recursiva dos parâmetros de Markov, $\mathbf{I}_{\mathbf{P}_{y u}^{i}} \in$ $\mathbb{R}^{[m+q(m+r)] \times[m+q(m+r)]}$ é a matriz identidade associada a $\mathbf{P}_{y u}^{i}(k-1)$ (Aguirre, 2015). 


\subsubsection{Algoritmo de realização de autossistemas (ERA)}

A partir dos parâmetros de Markov estimados $\underline{\widehat{\mathbf{Y}}}_{y u}^{i}(k)$, a estimação de cada submodelo local inverso, referente a regra $i$, é obtida pelo algoritmo de realização de autossistemas, ou simplesmente ERA (do inglês Eigensystems Realization Algorithm) (Juang, 1994; Chen, 1999), a fim de estimar submodelos de realização mínima de ordem reduzida na forma canônica do observador Wu et al. (2015), os quais são necessários para o mapeamento inverso do sistema dinâmico em cada ponto de operação. Assim, a matriz de Hankel, em função dos parâmetros de Markov, é dada por:

$$
\mathcal{H}_{y u}^{i}(\varkappa-2) \triangleq\left[\begin{array}{cccc}
\left(\widehat{\boldsymbol{\Upsilon}}_{y u}^{i}\right)_{\varkappa} & \left(\widehat{\boldsymbol{\Upsilon}}_{y u}^{i}\right)_{\varkappa+1} & \cdots & \left(\widehat{\boldsymbol{\Upsilon}}_{y u}^{i}\right)_{\varkappa+g-1} \\
\left(\widehat{\boldsymbol{\Upsilon}}_{y u}^{i}\right)_{\varkappa+1} & \left(\widehat{\boldsymbol{\Upsilon}}_{y u}^{i}\right)_{\varkappa+2} & \cdots & \left(\widehat{\boldsymbol{\Upsilon}}_{y u}^{i}\right)_{\varkappa+g} \\
\vdots & \vdots & \ddots & \vdots \\
\left(\widehat{\boldsymbol{\Upsilon}}_{y u}^{i}\right)_{\varkappa+p-2} & \left(\widehat{\boldsymbol{\Upsilon}}_{y u}^{i}\right)_{\varkappa+p-1} & \cdots & \left(\widehat{\boldsymbol{\Upsilon}}_{y u}^{i}\right)_{\varkappa+p+g-3} \\
\left(\widehat{\boldsymbol{\Upsilon}}_{y u}^{i}\right)_{\varkappa+p-1} & \left(\widehat{\boldsymbol{\Upsilon}}_{y u}^{i}\right)_{\varkappa+p} & \cdots & \left(\widehat{\boldsymbol{\Upsilon}}_{y u}^{i}\right)_{\varkappa+p+g-2}
\end{array}\right]
$$

$\forall \varkappa \geq 2$, onde $\mathcal{H}_{y u}^{i}(\varkappa-2) \in \mathbb{R}^{p r \times g(m+r)}$, e $p \geq 0$ e $g \geq 0$ são as contantes de observabilidade e controlabilidade, respectivamente, ambas inteiras e arbitrárias. Observa-se que $p r<g(m+r)$ e $p \geq q$ são condições necessárias para executar o ERA via matriz de Hankel (Juang, 1994; Wu et al., 2015). Logo, calcula-se $\mathcal{H}_{y u}^{i}(0)$ e $\mathcal{H}_{y u}^{i}(1)$, e em seguida realiza-se a operação de decomposição de valores singulares sobre $\mathcal{H}_{y u}^{i}(0)$, para obter a definição da ordem de realização mínima reduzida $n_{\min }$. Porém, dentre os $n$ valores singulares de $\mathcal{H}_{y u}^{i}(0)$, os $n-n_{\text {min }}$ valores singulares não significativos são aqueles relativamente pequenos, por conterem mais informações ruidosas do que do sistema (Juang, 1994; Wu et al., 2015). Assim, define-se um valor de $n_{\min } \leq n$ de tal forma que exclua tais valores singulares não significativos para todos os $c$ submodelos locais inversos.

Uma vez definido o valor de $n_{m i n}$, realiza-se a estimação paramétrica via $\mathcal{H}_{y u}^{i}(0)$ e $\mathcal{H}_{y u}^{i}(1)$. A priori, define-se as seguintes matrizes seletoras:

$$
\begin{aligned}
& \mathcal{E}_{r} \triangleq\left[\begin{array}{c}
\mathbf{I}_{r} \\
\mathbf{0}_{(p-1) r \times r}
\end{array}\right] \\
& \mathcal{E}_{n_{m i n}} \triangleq\left[\begin{array}{c}
\mathbf{I}_{n_{\text {min }}} \\
\mathbf{0}_{\left(p r-n_{m i n}\right) \times n_{m i n}}
\end{array}\right] \\
& \mathcal{E}_{m+r} \triangleq\left[\begin{array}{c}
\mathbf{I}_{m+r} \\
\mathbf{0}_{(g-1)(m+r) \times(m+r)}
\end{array}\right]
\end{aligned}
$$

tal que $\mathcal{E}_{r} \in \mathbb{R}^{p r \times r}, \mathcal{E}_{n_{m i n}} \in \mathbb{R}^{p r \times n_{m i n}}, \mathcal{E}_{m+r} \in$ $\mathbb{R}^{g(m+r) \times(m+r)} . \quad \mathbf{I}_{r} \in \mathbb{R}^{r \times r}, \mathbf{I}_{n_{\min }} \in \mathbb{R}^{n_{\min } \times n_{\min }} \mathrm{e}$ $\mathbf{I}_{m+r} \in \mathbb{R}^{(m+r) \times(m+r)}$ são matrizes identidade, enquanto que $\mathbf{0}_{(p-1) r \times r} \in \mathbb{R}^{(p-1) r \times r}, \mathbf{0}_{\left(p r-n_{\min }\right) \times n_{\min }} \in$ $\mathbb{R}^{\left(p r-n_{\text {min }}\right) \times n_{\text {min }}}$ e $\mathbf{0}_{(g-1)(m+r) \times(m+r)} \in \mathbb{R}^{(g-1)(m+r) \times(m+r)}$ são matrizes de zeros. Assim, os parâmetros $\widehat{\mathbf{G}}^{i}, \widehat{\mathbf{H}}^{i}, \widehat{\mathbf{O}}^{i}$, $\widehat{\boldsymbol{\Gamma}}^{i}$ e $\widehat{\boldsymbol{\Delta}}^{i}$ podem ser estimados da seguinte forma:

$$
\begin{aligned}
& \widehat{\mathbf{G}}^{i}=\mathcal{E}_{n_{m i n}}^{T} \mathcal{H}_{y u}^{i}(1) \\
& \cdot\left(\left[\mathcal{E}_{n_{\text {min }}}^{T} \mathcal{H}_{y u}^{i}(0)\right]^{T}\right. \\
& \left.\cdot\left\{\mathcal{E}_{n_{m i n}}^{T} \mathcal{H}_{y u}^{i}(0)\left[\mathcal{E}_{n_{m i n}}^{T} \mathcal{H}_{y u}^{i}(0)\right]^{T}\right\}^{-1}\right) \\
& {\left[\widehat{\mathbf{H}}^{i}, \widehat{\mathbf{O}}^{i}\right]=\mathcal{E}_{n_{m i n}}^{T} \mathcal{H}_{y u}^{i}(0) \mathcal{E}_{m+r}} \\
& \widehat{\mathbf{H}}^{i}=\text { as primeiras } m \text { colunas de } \\
& {\left[\widehat{\mathbf{H}}^{i}, \widehat{\mathbf{O}}^{i}\right] \text {. }} \\
& \widehat{\mathbf{O}}^{i}=\underset{\left[\widehat{\mathbf{H}}^{i}, \widehat{\mathbf{O}}^{i}\right] .}{\operatorname{arltimas}} r \text { colunas de } \\
& \widehat{\boldsymbol{\Gamma}}^{i}=\mathcal{E}_{r}^{T} \mathcal{E}_{n_{m i n}} \\
& \widehat{\boldsymbol{\Delta}}^{i}=\text { as primeiras } m \text { colunas de } \\
& \underline{\widehat{\mathbf{Y}}}_{y u}^{i}(k) \text {. }
\end{aligned}
$$

\section{RESULTADOS EXPERIMENTAIS}

Para demonstrar a aplicabilidade da metodologia proposta, os resultados experimentais da identificação nebulosa inversa de um helicóptero com dois graus de liberdade (do inglês Helicopter with 2 Degrees Of Freedoms - H2DOF) (Filho and de Oliveira Serra, 2017; Pires and Serra, 2018; Filho and de Oliveira Serra, 2018), são apresentados. O vetor de entradas do Helicóptero 2DoF, é definido como $\mathbf{u}=\left[u_{1}, u_{2}\right]^{T} \triangleq\left[V_{\theta}, V_{\psi}\right]^{T}$, com $V_{\theta} \in$ $[-24,24] \subset \mathbb{R}_{+}^{*}$ e $V_{\psi} \in[-15,15] \subset \mathbb{R}_{+}^{*}$ são níveis de tensão CC dos motores que proporcionam o torque nos eixos de elevação e azimute, dados em volts $(V)$ respectivamente, e o vetor de saídas é definido como $\mathbf{y}=\left[y_{1}, y_{2}\right]^{T} \triangleq[\theta, \psi]^{T}$, $\operatorname{com} \theta \in[-40,5,40,5] \subset \mathbb{R}_{+}^{*}$ e $\psi \in[0,360] \subset \mathbb{R}_{+}^{*}$ sendo as posições angulares de elevação e azimute, dados em graus $\left(^{\circ}\right)$, respectivamente. O Helicóptero 2DoF, em malha aberta, não atende ao critério de BIBO estabilidade. Assim, foram utilizados 1096 pontos como conjunto de dados, em malha aberta, amostrados a cada $T_{s}=3 \cdot 10^{-3}$ (s), com sinais pseudoaleatórios aplicados às entradas, de tal forma que os sinais das saídas não ultrapassem seus respectivos intervalos de operação. Além disso, desse conjunto de dados, foram separados os $l=365$ primeiros pontos para serem utilizados como estimação inicial em batelada; já os 731 pontos restantes foram usados para a etapa de estimação recursiva.

O modelo nebuloso inverso, descrito na metodologia, foi definido com $c=3$ regras. Para estimação da proposição antecedente, definiu-se os seguintes parâmetros de configuração do algoritmo GK: expoente de ponderação dos agrupamentos $\varrho=2$; tolerância de terminação das iterações de otimização $\vartheta=10^{-3}$; número de iterações de otimização $L=100$; volume do $i$-ésimo agrupamento $\nu_{i}=1$, para todo $i \in[1, c]$. Referente a $i$-ésima matriz de covariância $\mathbf{F}^{i}$, definiu-se os seguintes parâmetros: limite do número condicional $\alpha=10^{15}$ e o parâmetro de ponderação $\varsigma=0,5$. Já para a estimação recursiva 
por meio da versão nebulosa do algoritmo OKID, definiuse os seguintes parâmetros de configuração: constante de predição associado com os estados $q=1$; constante de observabilidade $p=10$, e constante de controlabilidade $g=5$, ambas associadas às matrizes de Hankel $\mathcal{H}_{y u}^{i}(0) \mathrm{e}$ $\mathcal{H}_{y u}^{i}(1)$; a constante de suavização exponencial $T_{f}=40$ e a ordem de realização mínima reduzida $n_{\text {min }}=2$. De acordo com a metodologia adotada, o modelo nebuloso inverso foi obtido, experimentalmente, a partir de duas etapas. Na primeira etapa, utilizou-se um conjunto de dados para estimação inicial, em batelada, das matrizes $\underline{\mathbf{Y}}_{y u}^{i}(k)$, dos parâmetros $\widehat{\mathbf{G}}^{i}(l), \widehat{\mathbf{H}}^{i}(l), \widehat{\mathbf{O}}^{i}(l), \widehat{\boldsymbol{\Gamma}}^{i}(l), \widehat{\boldsymbol{\Delta}}^{i}(l)$, dos vetores $\hat{\mathbf{x}}_{y u}^{i}(k), \hat{\mathbf{u}}^{i}(k)$, para a $i$-ésima regra, e das variáveis $\tilde{\hat{\mathbf{x}}}_{y u}(k)$, $\tilde{\hat{\mathbf{u}}}(k)$. Em seguida, a partir do modelo nebuloso inverso inicialmente parametrizado, cada submodelo linear inverso, para a $i$-ésima regra, foi parametrizado de forma recursiva, conforme formulação apresentada nas seções 2.2.1 e 2.2.2.

As estimações dos estados nebulosos inversos $\tilde{\hat{\mathbf{x}}}_{y u}(k)$ encontram-se ilustradas nas Figuras 1 e 2. Já as estimações das saídas $\tilde{\hat{\mathbf{u}}}(k)$ do modelo nebuloso inverso, em comparação com as entradas do Helicóptero 2DoF, encontram-se ilustrados nas Figuras 3 e 4 . As métricas utilizadas para avaliar a qualidade das estimações paramétricas recursivas, referentes ao modelo nebuloso inverso, e portanto o desempenho da metodologia adotada, foram o erro médio quadrático normalizado normalizado (do inglês Normalized Mean Squared Error - NMSE), e o índice quadrático de confiabilidade (ou coeficiente de determinação) $R^{2}$. Quanto mais próximo de 0 é o valor do NMSE e mais próximo de 1 é o valor do $R^{2}$, respectivamente, significa que mais exatas e precisas são as estimações. Nas Figuras 5 e 6 , encontram-se registrados as métricas supracitadas.

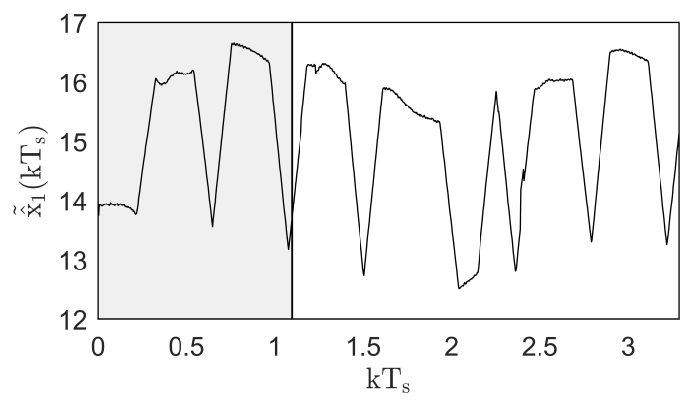

Figura 1. H2DOF: estado estimado do modelo nebuloso $\tilde{\hat{x}}_{1}\left(k T_{s}\right)$. Etapas: região $\square$ (estimação inicial), região $\square$ (estimação recursiva). Unidades: eixo $\mathrm{kT}_{\mathrm{S}}(s)$.

\section{CONCLUSÕES}

A metodologia adotada consistiu na formulação da identificação nebulosa inversa adaptativa com abordagem direta, no contexto do espaço de estados com observador de Kalman, a partir de dados experimentais. Analisando-se os resultados apresentados, pode-se concluir:

a) A metodologia proposta nesse artigo possibilita a estimação dos estados (nebulosos) inversos do sistema, o que proporciona o desenvolvimento de novas

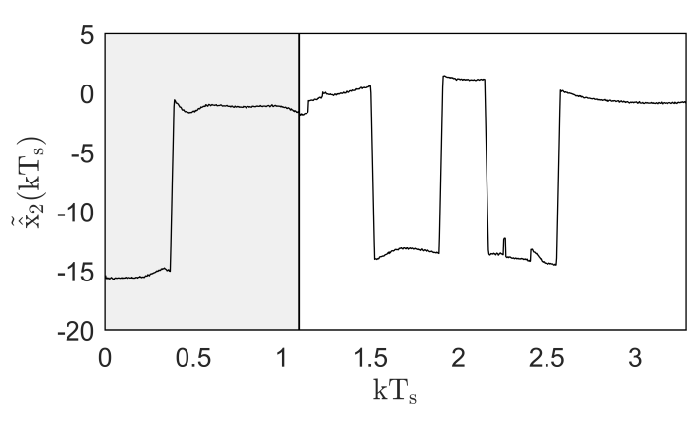

Figura 2. H2DOF: estado estimado do modelo nebuloso $\tilde{\hat{x}}_{1}\left(k T_{s}\right)$. Etapas: região $\square$ (estimação inicial), região $\square$ (estimação estimação recursiva). Unidades: eixo $\mathrm{kT}_{\mathrm{s}}(s)$.

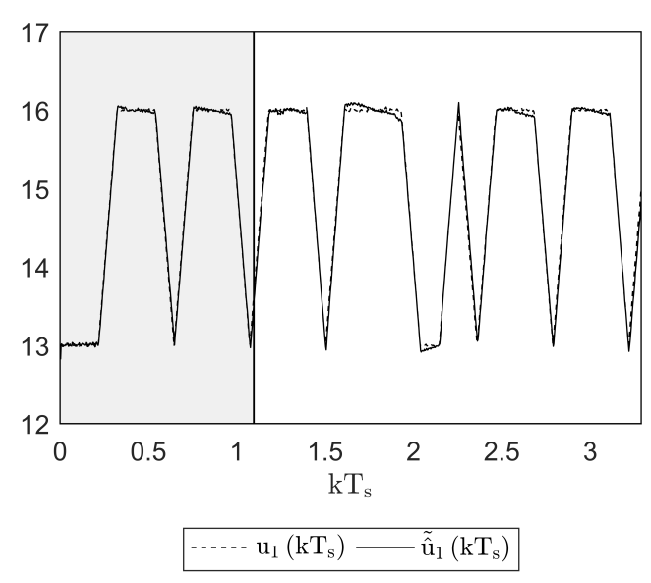

Figura 3. H2DOF: comparação entre a saída do modelo nebuloso $\tilde{\hat{u}}_{1}\left(k T_{s}\right)$ e a entrada do sistema $u_{1}\left(k T_{s}\right)$. Etapas: região $\square$ (estimação inicial), região $\square$ (estimação recursiva). Unidades: eixo $\mathrm{kT}_{\mathrm{s}}(s)$, eixos $\tilde{\hat{\mathrm{u}}}_{1}\left(\mathrm{kT}_{\mathrm{s}}\right)(V)$ e $\mathrm{u}_{1}\left(\mathrm{kT}_{\mathrm{s}}\right)(V)$.

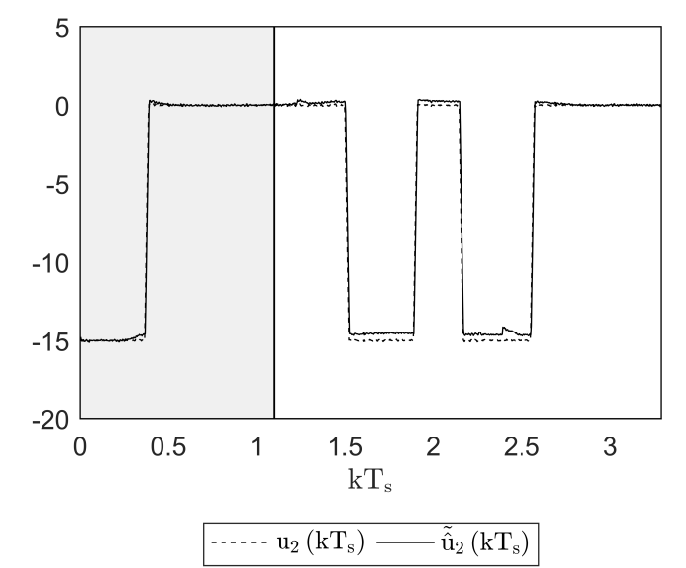

Figura 4. H2DOF: comparação entre a saída do modelo nebuloso $\tilde{\hat{u}}_{2}\left(k T_{s}\right)$ e a entrada do sistema $u_{2}\left(k T_{s}\right)$. Etapas: região $\square$ (estimação inicial), região $\square$ (estimação recursiva). Unidades: eixo $\mathrm{kT}_{\mathrm{s}}(s)$, eixos $\tilde{\hat{\mathrm{u}}}_{2}\left(\mathrm{kT}_{\mathrm{s}}\right)(V)$ e $\mathrm{u}_{2}\left(\mathrm{kT}_{\mathrm{s}}\right)(V)$. 


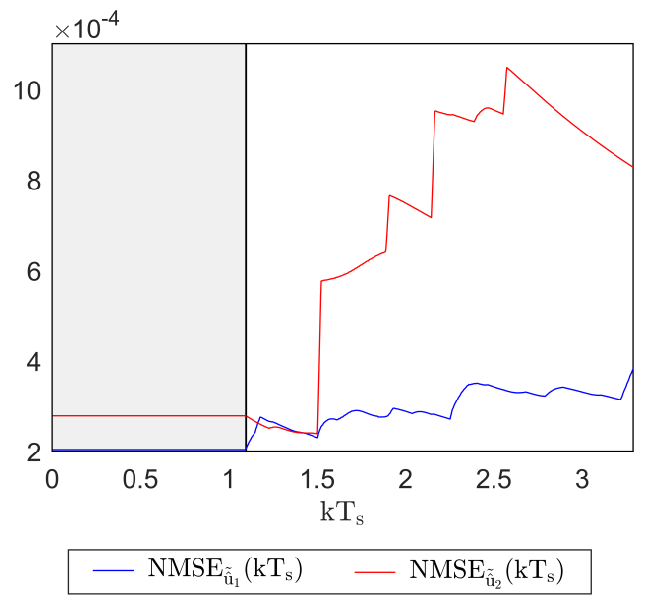

Figura 5. H2DOF: métrica do erro médio quadrático normalizado NMSE associada com as saídas do modelo nebuloso inverso $\tilde{\hat{\mathbf{u}}}=\left[\tilde{\hat{\mathrm{u}}}_{1}, \tilde{\hat{\mathrm{u}}}_{2}\right]^{T}$. Etapas: região $\square$ (estimação inicial), região $\square$ (estimação recursiva). Unidades: eixo $\mathrm{kT}_{\mathrm{s}}(s)$.

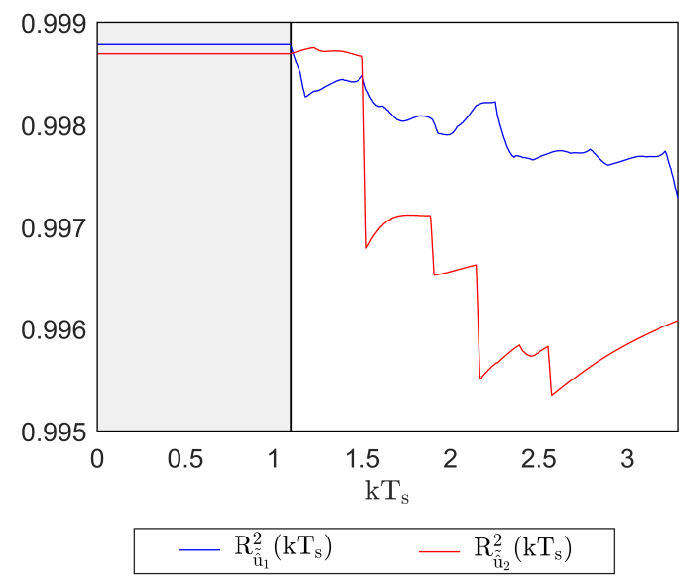

Figura 6. H2DOF: métrica confiabilidade $R^{2}$ associada com as saídas do modelo nebuloso inverso $\tilde{\hat{\mathbf{u}}}=\left[\tilde{\hat{\mathrm{u}}}_{1}, \tilde{\hat{\mathrm{u}}}_{2}\right]$. Etapas: região $\square$ (estimação inicial), região $\square$ (estimação recursiva). Unidades: eixo $\mathrm{kT}_{\mathrm{s}}(s)$.

estratégias de análise de comportamento inverso e de controle de sistemas e fenômenos físicos.

b) As tendências de otimalidade dos indicadores de qualidade NMSE e $R^{2}$ representam a eficiência da metodologia adotada quanto a sua aplicabilidade.

\section{REFERÊNCIAS}

Aguirre, L.A. (2015). Introdução à Identificação de Sistemas: Técnicas Lineares e Não-Lineares Aplicadas a Sistemas Reais. UFMG, Minas Gerais, 4 edition.

Babuška, R., van der Veen, P.J., and Kaymak, U. (2002). Improved covariance estimation for Gustafson-Kessel clustering. In Fuzzy Systems, 2002. FUZZ-IEEE'02. Proceedings of the 2002 IEEE International Conference on, volume 2, 1081-1085.

Babuška, R. (1998). Fuzzy Modeling for Control, volume 27. Kluwer Academic Publishers, Boston.
Boukezzoula, R., Galichet, S., and Foulloy, L. (2003). Nonlinear internal model control: Application of inverse model based fuzzy control. IEEE TRANSACTIONS ON FUZZY SYSTEMS, 11(6), 814-829.

Chen, C.T. (1999). Linear System Theory and Design. Oxford University Press, New York, 3rd. edition.

Filho, O.D.R. and de Oliveira Serra, G.L. (2017). Adaptive neuro fuzzy black box modeling based on instrumental variable evolving algorithm. Journal of Control, Automation and Electrical Systems, 28(1), 50-67.

Filho, O.D.R. and de Oliveira Serra, G.L. (2018). Recursive fuzzy instrumental variable based evolving neurofuzzy identification for non-stationary dynamic system in a noisy environment. Fuzzy sets and Systems, 338, 50-89.

Juang, J.N. (1994). Applied System Identification. Prentice Hall, Upper Saddle River, 4. th edition.

Jung, Y. and Enqvist, M. (2013). Estimating models of inverse systems. In 52nd Annual Conference on Decision and Control $(C D C)$, 52, 7143-7148. IEEE, Florence.

Lai, G., Liu, Z., Zhang, Y., Chen, C.L.P., Xie, S., and Liu, Y. (2017). Fuzzy adaptive inverse compensation method to tracking control of uncertain nonlinear systems with generalized actuator dead zone. IEEE Transactions on Fuzzy Systems, 25(1), 191-204.

Lee, M.H. and Liu, Y.W. (2014). Input load identification of nonlinear tower structural system using intelligent inverse estimation algorithm. Procedia Engineering, 79, 540-549.

Magalhães, A.M. and Serra, G.L.O. (2018). Proposta de identificação de sistemas dinâmicos não lineares baseada em modelo nebuloso takagi-sugeno inverso: Uma abordagem direta no contexto do espaço de estados. In XXII Congresso Brasileiro de Automática. João Pessoa. doi: 10.20906/CPS/CBA2018-1064. URL http://dx.doi. org/10.20906/CPS/CBA2018-1064.

Nelles, O. (2001). Nonlinear System Identification: from classical approaches to neural networks and fuzzy models. Springer, Verlag.

Pires, D. and Serra, G. (2018). An approach for fuzzy kalman filter modeling based on evolving clustering of experimental data. Journal of Intelligent \& Fuzzy Systems, 35(2), 1819-1834.

Rahbari, O., Omar, N., Firouz, Y., Rosen, M.A., Goutam, S., Bossche, P.V.D., and Mierlo, J.V. (2018). A novel state of charge and capacity estimation techinique for electric vehicles connected to a smart grid based on inverse theory and a metaheuristic algorithm. Energy, 155, 1047-1058.

Sousa, J.M.C. and Kaymak, U. (2002). Fuzzy Decision Making in Modeling and Control, volume 27. World Scientific, New Jersey. World Scientific Series in Robotics and Intelligent Systems.

Sun, C., Mu, C., and Li, X. (2009). A weighted LS-SVM approach for the identification of a class of nonlinear inverse system. Science in China Series F: Information Sciences, 52(5), 770-779.

Wu, C.Y., Tsai, J.S.H., Guo, S.M., Shieh, L.S., Canelon, J.I., Ebrahimzadeh, F., and Wang, L. (2015). A novel on-line observer kalman filter identification method and its application to input-constrained active fault-tolerant tracker design for unknown stochastic system. Journal of the Franklin Institute, 352, 1119-1151. 